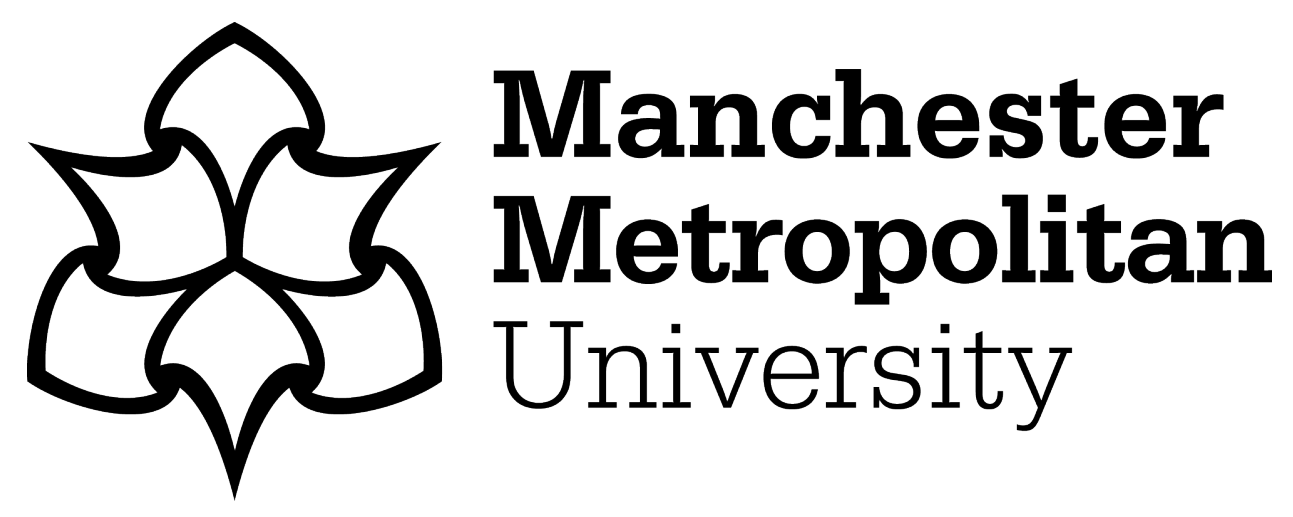

Lynch, S, Ramos, J, Jones, DA and Degens, H (2017) Hysteresis in muscle. International Journal of Bifurcation and Chaos, 27 (1). p. 1730003. ISSN 0218-1274

Downloaded from: https://e-space.mmu.ac.uk/617562/

Version: Accepted Version

Publisher: World Scientific Publishing

DOI: https://doi.org/10.1142/S0218127417300038

Please cite the published version 


\title{
HYSTERESIS IN MUSCLE
}

\author{
Jorgelina Ramos \\ School of Healthcare Science, \\ Manchester Metropolitan University, Chester St., Manchester M1 5GD, \\ United Kingdom, \\ j.ramos@mmu.ac.uk \\ Stephen Lynch \\ School of Computing, Mathematics and Digital Technology, Manchester \\ Metropolitan University, Chester St., Manchester M1 5GD, United \\ Kingdom, \\ s.lynch@mmu.ac.uk \\ David Jones \\ School of Healthcare Science, \\ Manchester Metropolitan University, Chester St., Manchester M1 5GD, \\ United Kingdom, \\ jonesda14@gmail.com \\ Hans Degens \\ School of Healthcare Science, \\ Manchester Metropolitan University, Chester St., Manchester M1 5GD, \\ United Kingdom, and Lithuanian Sports University, Kaunas, Lithuania. \\ h.degens@mmu.ac.uk
}

This paper presents examples of hysteresis from a broad range of scientific disciplines and demonstrates a variety of forms including clockwise, counterclockwise, butterfly, pinched and kiss-andgo, respectively. These examples include mechanical systems made up of springs and dampers which have been the main components of muscle models for nearly one hundred years. For the first time, as far as the authors are aware, hysteresis is demonstrated in single fibre muscle when subjected to both lengthening and shortening periodic contractions. The hysteresis observed in the experiments is of two forms. Without any relaxation at the end of lengthening or shortening, the hysteresis loop is a convex clockwise loop, whereas a concave clockwise hysteresis loop (labeled as kiss-and-go) is formed when the muscle is relaxed at the end of lengthening and shortening. This paper also presents a mathematical model which reproduces the hysteresis curves in the same form as the experimental data.

Keywords: Bistability, critical slowing down, feedback, Hill's mathematical model, hysteresis, muscle single fibre, MATLAB 


\section{Introduction}

In 1885, Sir James Alfred Ewing first coined the term hysteresis whilst showing the persistent effects on ferric metals exposed temporarily to magnetic fields [Ewing, 1885]. The irreversibility of the magnetisation and demagnetisation processes makes these hysteretic devices useful as magnetic memory, and indeed, the magnetic memory characteristics of chromium and iron oxides has led to the continued development of magnetic storage media used to store computer data as well as audio and video signals [Piramanayagam \& Chong, 2011].

Hysteresis is the time-based dependence of a system's output based on present and past outputs. In some applications, the input-output diagram is the same at every frequency. Such systems have rate independent hysteresis. However, in most systems the dynamic response changes with the input frequency giving distinct diagrams for different frequencies of excitation. Therefore, these systems have rate dependent hysteresis [Muller \& Xu, 1991]. As muscle tires with repeated use it is not surprising to find that hysteresis in muscle is rate dependent.

The two essential ingredients for hysteresis are nonlinearity and feedback. Hysteresis is not a new phenomenon, indeed it abounds throughout the realms of science. Hysteresis has its foundations in physics, in elasticity [Muller \& Xu, 1991], in ferroelectric and ferromagnetic materials [Smith et al., 2006; Damjanovic, 2005] and Lynch et al. have demonstrated hysteresis in a wide range of nonlinear optical resonators and microfiber ring resonators [Lynch \& Steele, 2011; Lynch et al., 2015]. In electric circuits, Borresen and Lynch [2002], have demonstrated hysteresis in Chua's electrical circuit for the first time. In mechanical engineering, hysteresis is possible between multiple stable limit cycles [Lynch \& Christopher, 1999] when investigating surge in jet engines and wing rock phenomena in modern aircraft. In chemical kinetics, simple models of Hopf bifurcation in a Brusselator model and hysteresis in an autocatalytic chemical reaction are demonstrated [Lynch, 2011]. In economics, hysteresis is present in inflation-unemployment models [Ball, 2009]. In biology, there is hysteresis in blood cell population dynamics [Lynch, 2005], muscle cross-bridge models [Walcott, 2009] and neuronal networks [Lynch \& Bandar, 2005], and hysteresis is present in cell biology [Pomenering et al., 2003], genetics [Kramer \& Fussenegger, 2005], immunology [Das et al., 2009], angiogenesis and haematopoiesis [Lynch \& Borresen, 2015] and Noori [2014], covers more general examples of hysteresis phenomena in biology.

Many nonlinear dynamical systems display hysteresis when some form of feedback mechanism is present. When modeling systems mathematically, bifurcation diagrams are plotted in order to display the hysteresis phenomena using packages such as MATLAB ${ }^{\mathbb{Q}}$, Maple ${ }^{\mathrm{TM}}$ and Mathematica ${ }^{\mathbb{Q}}$ [Lynch, 2014, $2010,2007]$. The second iterative method is adopted where a parameter is varied and the solution to the previous iterate is used as the initial condition for the next iterate. In this case, there is a history associated with the process and only one point is plotted for each value of the parameter. For example, most of the bifurcation diagrams plotted in this section were plotted using the second iterative method. The system has to have at least two stable steady states which could be fixed points, critical points or stable limit cycles.

The different forms of hysteresis will now be illustrated by means of example.

\subsection{Biological hysteresis}

\subsubsection{Agar gel}

For most biologists, if not all, agar is a well-known gelling agent in solid media used commonly in microbiology. It is defined as a hydrophilic colloid that contains two major fractions, agarose, which is the gelling fraction, and the non-gelling fraction agaropectin. Agar is found as the supporting structure of the cell walls in a certain species of algae and the interest for this study is the characteristic "gelation hysteresis", as shown in Figure 1, between the melting and setting temperature. Typically, agar gels at temperatures between $32^{\circ}$ and $45^{\circ}$ when cooled, depending on the used seaweed, and needs to be heated above $90^{\circ}$ to melt and form a good solution [Armisen \& Galatas, 1987; Lahaye \& Rochas, 1991]. 


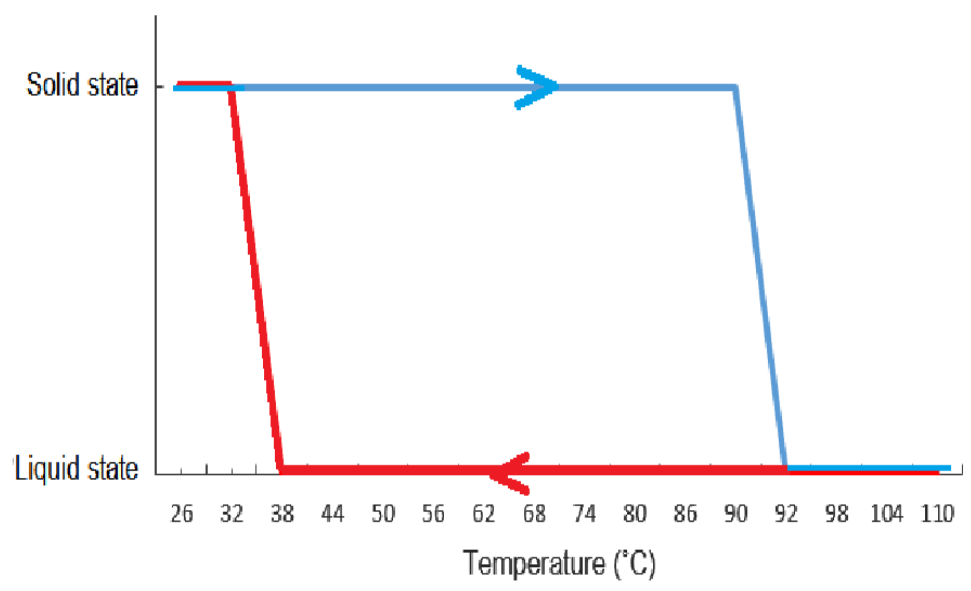

Fig. 1. Clockwise hysteresis cycle between the melting and setting temperatures, 32 and $90^{\circ}$, respectively, of the agar gel. Whether the agar is solid or liquid at a certain temperature between the melting and gelling states will depend on the previous state of the substance.

\subsection{Mechanical hysteresis}

\subsubsection{The preloaded two-bar linkage mechanism}

Historically, muscle has been modeled using springs and dampers and so this section includes those type of models. Drincic and Berstein [2011], analysed the dynamics of a two-bar linkage mechanism with joints $P, Q$ and $R$, preloaded by a stiffness $k$, as shown in Figure 2. A periodic force $F$ is applied at $Q$, where the two bars are joined by a frictionless pin. The angle $\theta$ denotes the counterclockwise angle the left bar makes with the horizontal, $q$ denotes the distance between $P$ and $R$, and $x$ is the distance between the joint $Q$ and the horizontal dashed line shown in Figure 2.

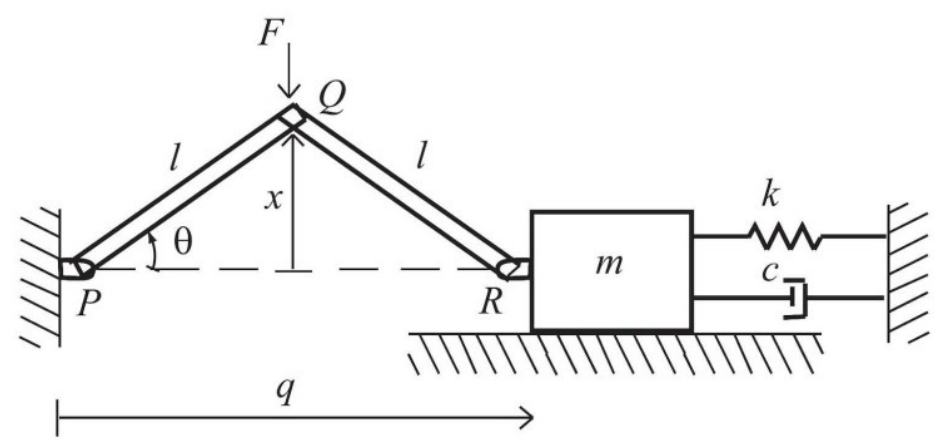

Fig. 2. The preloaded two-bar linkage with a periodic force $F$ acting at the joint $Q$. As the point $Q$ moves vertically up and down, the mass $m$ moves horizontally left and right.

Then,

$$
\begin{gathered}
x=I \sin \theta \\
q=2 I \cos \theta \\
F=\sin (w t) .
\end{gathered}
$$

The equations of dynamics for the preloaded two-bar linkage are given by 


$$
\begin{aligned}
\left(\left(2 m l^{2}+\right.\right. & \left.\left.\frac{9}{8} m_{b a r} l^{2}\right) \sin ^{2} \theta+\frac{5}{24} m_{b a r} l^{2}\right) \ddot{\theta}+\left(2 m l^{2}+\frac{9}{8} m_{b a r} l^{2}\right) \sin \theta \cos \theta \dot{\theta}^{2} \\
& +2 c l^{2} \sin ^{2} \theta \dot{\theta}+2 k I^{2}\left(\cos \theta_{0}-\cos \theta\right) \sin \theta=-\frac{I \cos \theta}{2} F
\end{aligned}
$$

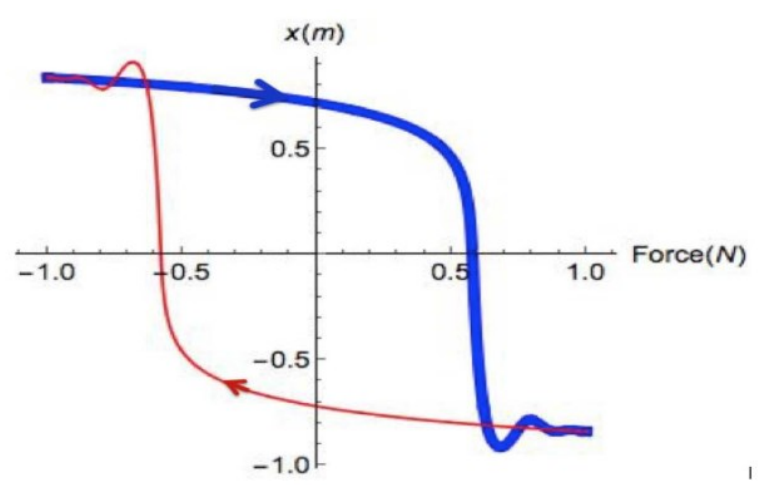

Fig. 3. Input-output clockwise hysteresis loop between $F$ and $x$ at $\omega=0.05$ radians per second. The area of the loop is the energy dissipated in a complete cycle and is proportional to the frequency of stimulation. The lower the frequency, the lower the dissipated energy, thus, the smaller the area of the loop. Note the ringing at the ends of the hysteresis loop, a common occurrence in hysteresis. The parameters used are $\mathrm{k}=1(\mathrm{~N} / \mathrm{m}), \mathrm{m}=1(\mathrm{~kg}), \mathrm{c}=1(\mathrm{Ns} / \mathrm{m}), \mathrm{m}_{\text {bar }}=0.5(\mathrm{~kg}), \mathrm{I}=1(\mathrm{~m})$ and $F=\sin (\omega t)$. As with other hysteresis loops shown in this section, the blue curve represents ramp up, in this case $F$ increase from $F=-1$ to $F=1$, and the red curve represents ramp down, where $F$ decreases from $F=1$ to $F=-1$.

and the dynamics in terms of the displacement q, using equations (1) to (4) is given by

$$
\begin{aligned}
& \left(\left(m+\frac{9}{16} m_{b a r}\right)\left(4 I^{2}-q^{2}\right)+\frac{5}{12} m_{b a r} I^{2}\right)\left(4 I^{2}-q^{2}\right) \ddot{q}+\frac{5}{12} m_{b a r} I^{2} q \dot{q}^{2} \\
& +c \dot{q}\left(4 I^{2}-q^{2}\right)^{2}+k\left(q-q_{0}\right)\left(4 I^{2}-q^{2}\right)^{2}=\frac{1}{2} q\left(4 I^{2}-q^{2}\right)^{\frac{3}{2}} F
\end{aligned}
$$

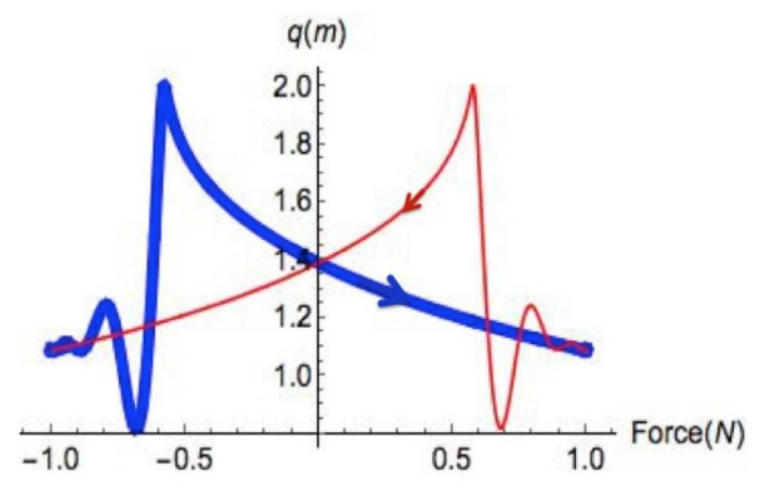

Fig. 4. Input-output map between the vertical force, $\mathrm{F}$, and the horizontal displacement, $\mathrm{q}$, given by equation (5) at a low frequency $\omega=0.05(\mathrm{r} / \mathrm{s})$. Note the ringing at the ends of the hysteresis loop. The parameters used are $\mathrm{k}=1(\mathrm{~N} / \mathrm{m}), \mathrm{m}=1$ $(\mathrm{kg}), \mathrm{c}=1(\mathrm{Ns} / \mathrm{m}), \mathrm{m}_{\mathrm{bar}}=0.5(\mathrm{~kg}), \mathrm{I}=1(\mathrm{~m})$ and $\mathrm{F}=\sin (\omega \mathrm{t})$.

Equations (4) and (5) represent the linkage dynamics under a periodic external force $F$. As in [Drincic \& Bernstein, 2011], take parameter values $\mathrm{k}=1(\mathrm{~N} / \mathrm{m}), \mathrm{m}=1(\mathrm{~kg}), \mathrm{c}=1(\mathrm{Ns} / \mathrm{m}), \mathrm{m}_{\mathrm{bar}}=0.5(\mathrm{~kg})$ and $\mathrm{I}=1(\mathrm{~m})$. Figure 3 shows the hysteresis loop for the vertical force $\mathrm{F}$, measured in Newtons, against the 
vertical displacement $x$, measured in metres. This figure demonstrates a clockwise hysteresis loop. Note the ringing at either end of the hysteresis loop where the steady state oscillates before settling on to a stable critical point. This ringing phenomenon is a standard feature with hysteresis loops. Note also that the area bounded by the hysteresis loop represents the energy dissipated in one cycle and is proportional to the frequency of stimulation.

Figure 4 shows the hysteresis loop for the vertical force F, measured in Newtons, against the horizontal displacement $\mathrm{q}$. This hysteresis loop has been labeled a butterfly hysteresis.

\subsubsection{The periodically forced nonlinear pendulum}

The Duffing equation can be used to model different physical systems [Thompson, 2002; Ott, 2002]. Here it is used to model a pendulum that is periodically driven and it has a nonlinear restoring force [Thompson, 2002]. A diagram of this system is presented in Figure 5.

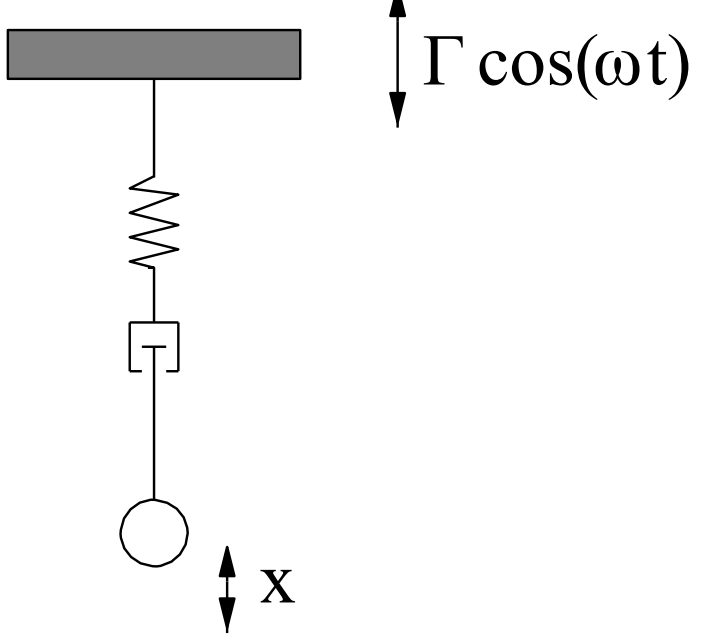

Fig. 5. Periodically forced pendulum with a cubic spring. The driving force is given by $F=\Gamma \cos (\omega t)$ and $x$ represents the displacement from equilibrium.

The equation modeling this system is given by

$$
\ddot{x}+k \dot{x}+\beta x+\alpha x^{3}=\Gamma \cos (\omega t)
$$

where $k \geq 0$ is the damping coefficient, $\beta$ is the stiffness, $\alpha$ is the nonlinear stiffness parameter, $\dot{x}$ is the speed of the mass, $\Gamma$ is the amplitude of force vibration and $\omega$ is the frequency of the driving force. When $\beta>0$, the Duffing equation models a periodically forced pendulum as depicted in Figure 5 . When $\alpha>0$, the spring is called a hardening spring and when $\alpha<0$, the spring is called a softening spring. When $\beta<0$, the Duffing equation models a periodically forced steel beam deflected between two magnets [Ott, 2002]. Consider the Poincaré map of system (6) as the amplitude $\Gamma$ varies when $k, \beta, \alpha$ and $\omega$ are fixed. The radius of the limit cycle on the Poincaré section is given by $r$. Figure 6 shows the hysteresis loop for a nonlinear pendulum when $\mathrm{k}=0.1, \beta=1, \alpha=0.1$, and $\omega=1.25$ and the forcing amplitude is ramped up from $\Gamma=0$ to $\Gamma=1$ and then ramped back down again. There is a counterclockwise hysteresis loop and the steady states in this case are both stable limit cycles. The examples illustrating wing rock and surge in jet engines [Lynch \& Christopher, 1999] also involve multiple steady states made up of stable limit cycles.

Chaotic phenomena in human-like reaching movements using a two-link arm mechanism driven by six muscles is described in [Rahatabad et al., 2011], where bifurcation maps, Lyapunov exponents and power spectra are employed to detect the chaos. In 1994, Webber and Zbilut applied recurrence plot strategies to assess physiological states such as respiration and muscle fatigue dynamically. The methodology is applicable to any rhythmical system including chemical, electrical, hormonal, neural and mechanical, and 


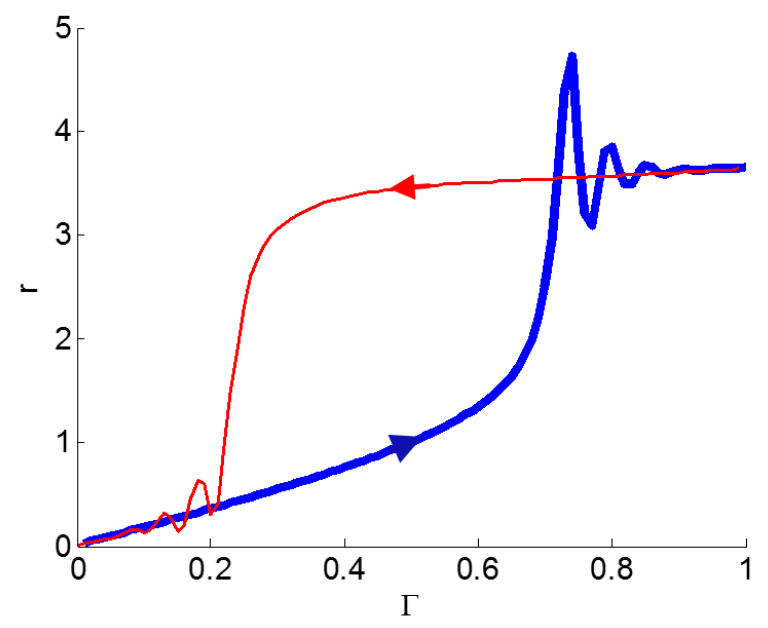

Fig. 6. Plot of $\Gamma$ versus $r$ for the nonlinear pendulum. Note the ringing at the ends of the hysteresis loop. The steady states in this case are stable limit cycles. The parameters used in this case were $k=0.1, \beta=1, \alpha=0.1$, and $\omega=1.25$, where $\Gamma$ is linearly ramped up from $\Gamma=0$ to $\Gamma=1$, and then ramped back down again.

provides a nonlinear diagnostic tool in assessing physiological systems and states. Chaos and chaos control in cardiac muscle has been extensively investigated, see [Weiss et al., 1999] and [Gauthier et al., 2002], in terms of heart rate variability and arrhythmic mortality, and practical applications of chaos theory to the modulation of human ageing were proposed by Kyriatzis et al. in 2003. A simple example of a system displaying hysteresis affected by chaos is now described.

\subsection{Optical hysteresis and chaos}

Optical hysteresis, or optical bistability, as it is sometimes known, was first proposed by Szöke et al. in 1969. There has been an immense amount of interest in it regarding its potential applications in high-speed alloptical signal processing, all-optical computing and more recently, optical sensing [Lynch \& Steele, 2011]. For optical hysteresis, nonlinearity is provided by the medium as a refractive (or dispersive) or absorptive nonlinearity, or both. The feedback is introduced through mirrors or fibre loops. A block diagram of the Simple Fibre Ring (SFR) resonator is shown in Figure 7.

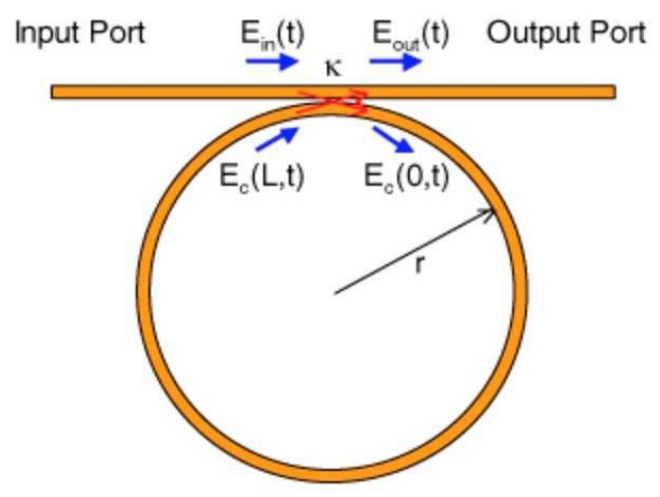

Fig. 7. A schematic of the SFR resonator. The electric fields entering and leaving the fibre ring are indicated. The parameter $K$ represents the power-splitting ratio at the coupler and $L$ is the length of the fibre loop.

Three different forms of hysteresis, namely, clockwise, counter-clockwise and butterfly, are all displayed in one system when modelling a homogeneously broadened two-level atomic system in a ring cavity within and without the rotating wave approximation [Lynch et al., 2015]. By considering the famous Maxwell- 
Bloch and Maxwell-Debye equations, Ikeda [Ikeda, 1979; Ikeda et al., 1980] theoretically investigated a nonlinear absorbing medium containing two-level atoms situated in a bulk ring cavity. He was able to demonstrate that optical circuits exhibiting optical hysteresis could also contain temporal instabilities, or chaos. Subject to certain constraints Ikeda was able to demonstrate that the complicated delay differential equations could be approximated by a much simpler discrete complex system [Lynch, 2014]. What's more, this simple system also accurately models the SFR [Lynch et al., 1998]. The iterative equation is given by

$$
E_{n+1}=A+B E_{n} e^{i\left|E_{n}\right|^{2}}
$$

where $A=i{ }_{\overline{1}} E_{i n}, B=V_{\bar{K}}$ and $E_{j}$ is the electric field amplitude at the $\mathbf{j}_{\text {th }}$ circulation around the fibre loop. A bifurcation diagram for system (7) is shown in Figure 8, where a counter-clockwise loop is clearly visible. Note that, to the right of the hysteresis loop is a period doubling cascade to chaos. As the parameter $B=\bar{K}$ increases, and more light is allowed to circulate in the fibre loop, the chaos shifts to the left and swamps the hysteresis loop, which should be avoided for applications.
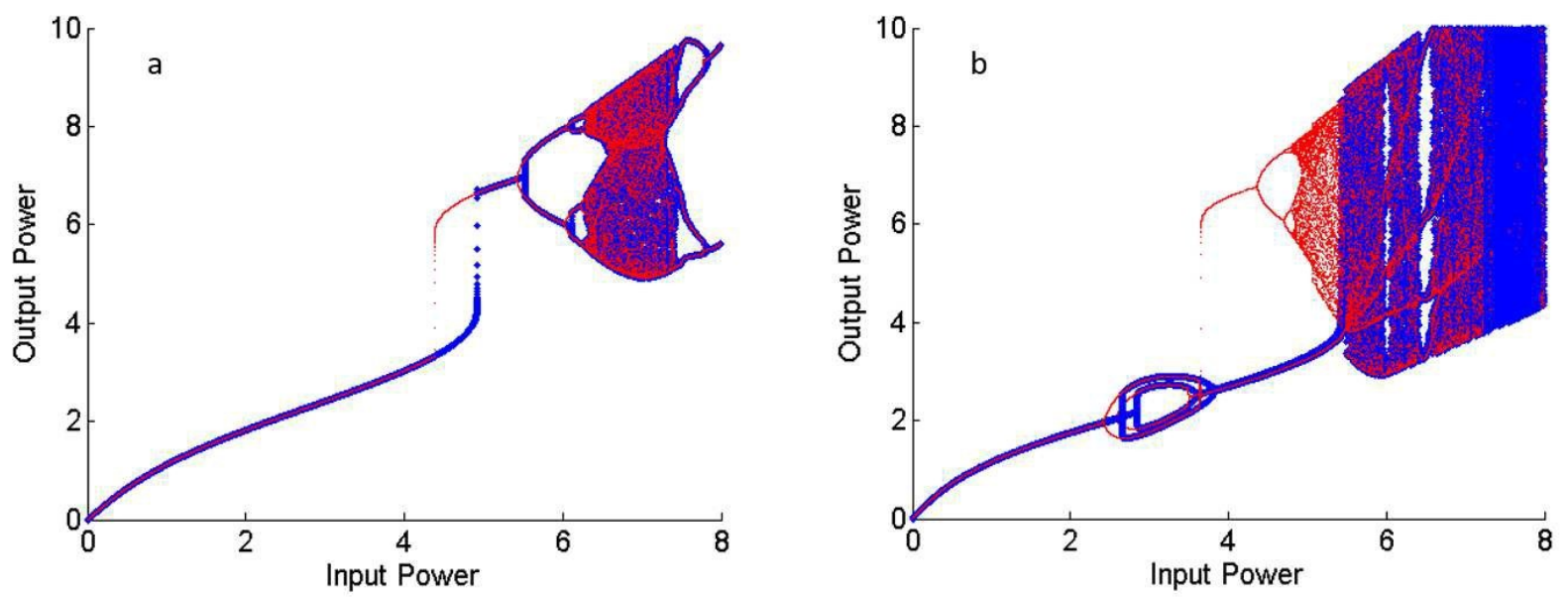

Fig. 8. Bifurcation diagram of the SFR resonator, the power $\mid A P^{2}$ was ramped up from $0 \mathrm{~W} \mathrm{~m}^{-2}$ to $8 \mathrm{~W} \mathrm{~m} \mathrm{~m}^{-2}$, and back down again. (a) When $B=0.15$, the isolated counter-clockwise hysteresis loop (bistable region) is followed by a region of period-doubling to chaos. (b) When $B=0.24$, instabilities encroach upon the hysteresis cycle at both ends.

\subsection{Memristors and pinched hysteresis}

Professor Leon Chua is widely acknowledged as the father of nonlinear circuit theory. For many decades it was believed that there are three fundamental electronic components, the resistor, the inductor and the capacitor, and many biological systems, such as the famous Hodgkin-Huxley system used to model the giant squid axon [Hodgkin \& Huxley, 1952], were simulated using these components. It is now known that there is another fundamental circuit element, and its sub-elements [Qingjiang et al., 2014], called the memristor. Chua [1971], used mathematics to prove the existence of the fourth nonlinear fundamental nonlinear element which relates flux and charge and he called this component the memristor. In 1976, Chua and Kang discovered that a memristor displays a pinched hysteresis, which is shown in Figure 9. It is now understood that memristors are a common phenomena in the living world as they are also seen in plants and invertebrates [Prodromakis et al., 2012]. More recently, Chua [2013] was able to correct a mistake in the Hodgkin-Huxley equations by introducing a memristor term in to their equations.

Readers interested in the mathematical analysis of the hysteresis models considered in the introductory section should consult the papers listed in the reference section.

Section 2 is concerned with the material and methods for the physical experimentation and Section 3 presents the experimental results. A mathematical model of lengthening and shortening muscle is presented in Section 4 and a discussion and further research are considered in Section 5. 

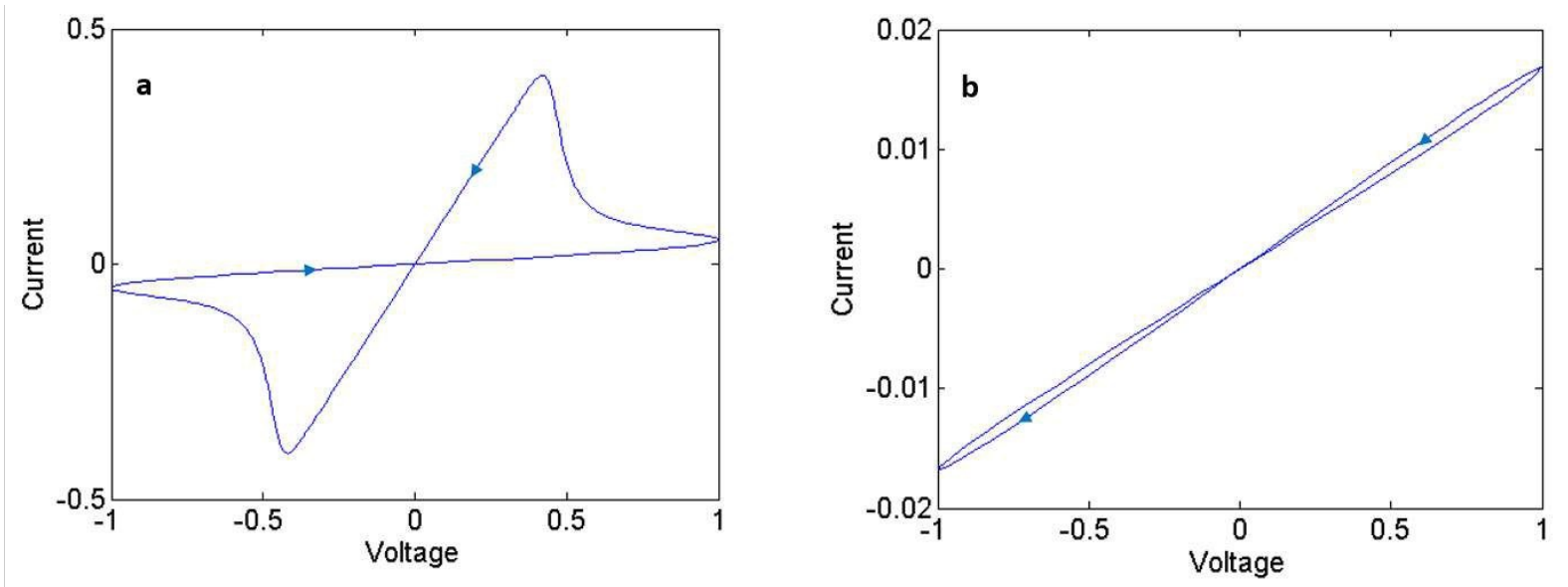

Fig. 9. Pinched hysteresis of a memristor in the $\mathbf{I}-\mathrm{V}$ plane. a For low frequencies there is a definite pinched hysteresis. $\mathrm{b}$ As the frequency gets higher, the pinched hysteresis degenerates and the memristor acts like a linear resistor. For more details see [Lynch, 2014].

\section{Materials and methods}

\subsection{Muscle samples}

Soleus muscles were dissected from 18-month-old (adult male, gtf2ird2) mice. The animals were humanely killed using approved schedule 1 methods (cervical dislocation) for other research projects approved by the local Animal Ethics Research Committee of the University of Manchester. This is in accord with the generally accepted guideline of reducing animal numbers to a minimum in biomedical research. The right soleus muscle was rapidly excised, weigh and immersed in glycerol/relax solution at $4^{\circ}$ for 24 hours. It was then treated with increasing concentrations of sucrose [Degens et al., 2010], which acts as an effective cryoprotectant, preventing damage of the contractile properties of the fibres. After sucrose-treatment, muscles were frozen in liquid nitrogen and stored at $-80^{\circ}$ for later use.

\subsection{Solutions}

The composition of the solutions have been described previously [Larsson \& Moss, 1993; Giliver et al., 2009; Degens et al., 2010]. The relaxing solution contained (mM): MgATP, 4.5; free Mg2+, 1; imidazole, 10; EGTA, 2 and $\mathrm{KCl}, 100$ and the $\mathrm{pH}$ was adjusted to 7.0 using $\mathrm{KOH}$. The glycerol/relax was the same as relaxing solution containing $50 \%(\mathrm{v} / \mathrm{v})$ glycerol. The $\mathrm{pCa}(-\log [$ free $\mathrm{Ca} 2+])$ of the activating solution was 4.5 and contained: MgATP, 5.3; free Mg2+, 1; imidazole, 20; EGTA, 7; creatine phosphate, 19.6; KCl, 64 with $\mathrm{pH} 7.0$.

\subsection{Preparation of single fibres}

The procedures for preparing single fibres have been described previously [Degens et al., 2010; Gilliver et al., 2011]. Briefly, fibre bundles were taken from the $-80^{\circ}$ storage, thawed and treated with decreasing concentrations of sucrose and stored in glicerol/relax at $-20^{\circ}$ for use within a month. The day of experiments, a small bundle was cut from the muscle and immersed in relaxing solution containing $1 \%$ Triton X-100 for 20 minutes to permeabilize the membranes and sarcoplasmic reticulum. After this, single fibres were teased from one end of the bundle and mounted in a permeabilized-fibre test system (400 Aurora Scientific Inc. Ontario, Canada). With the fibre mounted and immersed in relaxing solution, it was tied with nylon thread to fine insect pins attached to a force transducer (Aurora, 403) and a lever motor arm (Aurora, 312). The sarcomere length (sl) of the resting fibre was set at $2.6 \mu \mathrm{m}$ and checked along the length of the fibre. Fibre length (lo) was determined and checked at regular intervals thereafter. Fibre diameter was measured at three places along the length of the fibre while submerged in relaxing solution and the cross-sectional area calculated assuming the fibre to have a circular cross-section [Gilliver et al., 2010]. All experiments 
were carried out at $15^{\circ}$.

\subsection{Methods}

Fibres were transferred from the relaxing to the activating solution (pCa 4.5). When the isometric force (Fo) reached a plateau, the fibre was subjected to length changes following a bipolar triangle input with an amplitude of $5 \%$ at a frequency of $0.25\left(\mathrm{~s}^{-1}\right)$. A set of five fibres, one at a time, was used for this type of protocol. Then, another set of 20 fibres, one at a time, was subjected to the same wave with an extra resting time between the positive and negative half. The amplitudes for this second type of input were 3 $\%$ or $5 \%$ of lo at different frequencies starting from $f=0.125\left(\mathrm{~s}^{-1}\right)$ up to $1.25\left(\mathrm{~s}^{-1}\right)$.

Examples of the length change inputs and their corresponding force outputs are shown in Figure 10, where Fo and lo are the initial values of force and length respectively. The positive half of the input wave corresponded to stretches of either $3 \%$ or $5 \%$ of lo after which the fibre was returned to lo. On the other hand, the negative half corresponded to shortening contractions of the fibre, by the same percentages.
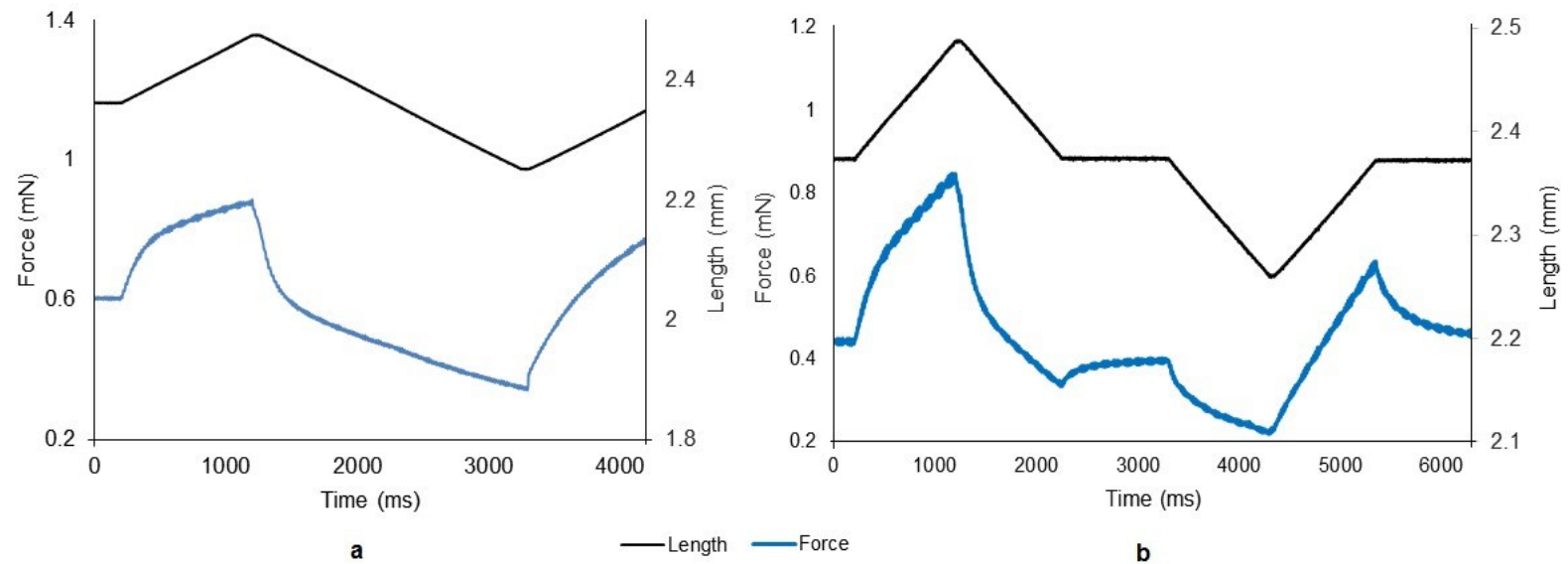

b

Fig. 10. Force response (blue curve) to a change in the fibre length (black curve). In case a, there is no rest between stretch and contraction, while in case $b$ there are short rest periods. The amplitude of the length change in both examples is $5 \%$ of lo, and the frequency is $f=0.25\left(s^{-1}\right)$ for $a$ and $f=0.167\left(s^{-1}\right)$ for $b$.

\section{Experimental results}

\subsection{Force response to a $5 \%$ lo length change}

In this section, the force-length relationship is plotted for a set of trials. It must be noted that in all the figures to follow, the origin of coordinates is where $\mathrm{F}=\mathrm{Fo}$ and $\mathrm{I}=\mathrm{l}$. The absolute values of these variables are different between fibres, therefore the origin is indicated in each figure. The response that appears in Figure 11 is a clockwise hysteresis loop representing the force-length relationship to one period of the bipolar triangle input shown in Figure 10a. Similar hysteresis loops were obtained with other fibres when there is no rest period.

When a relaxation time after each half wave was added, the response showed a concave hysteresis loop, as shown in Figure 12. These loops resemble those of kiss-and-go hysteresis [Volkov et al., 2014], which tend to touch each other at the center. The arrows indicate the clockwise direction of the loops.

For the rest of the figures, the force response is normalized as a percentage of Fo and a relaxation is imposed at the end of each lengthening and shortening interval. Similar figures to those shown in this paper can be plotted when there is no relaxation at the end of lengthening and shortening but the overall effect is the same in both cases.

In Figure 13, force vs length is plotted for two different fibres (a and b), stimulated at the same frequency. The response of another two single fibres (Figures 14a and 14b) was obtained at a higher 

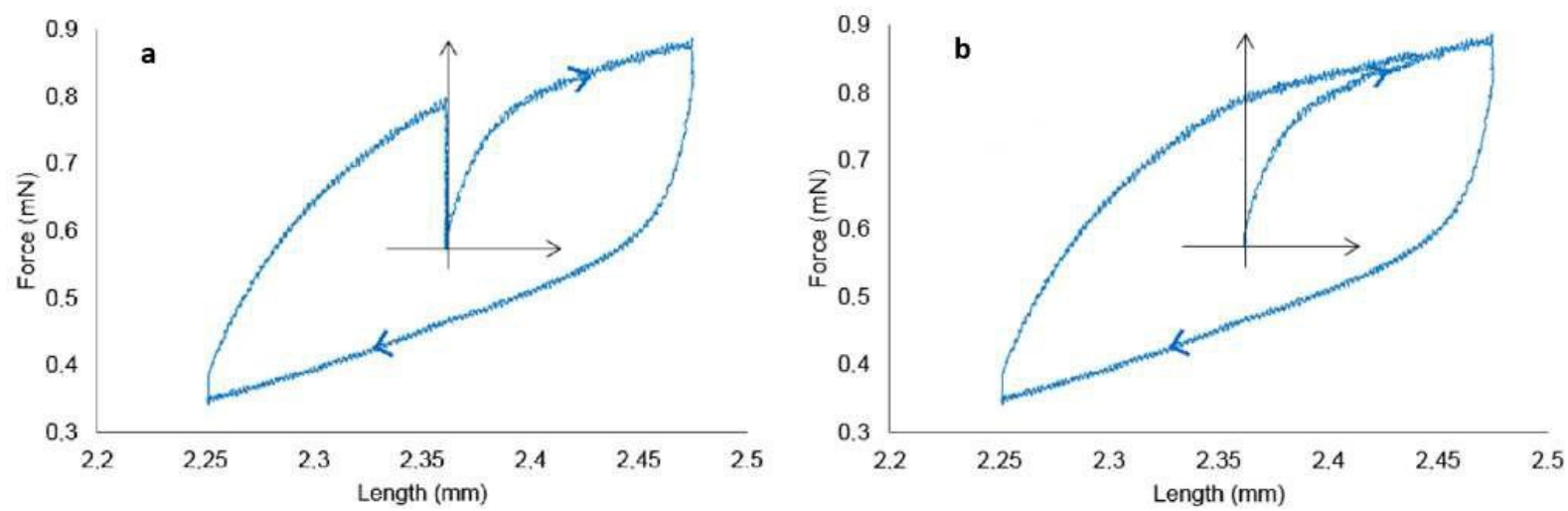

Fig. 11. a Force-length relationship corresponding to Figure 10a. The curve is a hysteresis loop for only one period of the input and for this fiber the origin is where $F_{0}=0.58(\mathrm{mN})$ and $l o=2.36(\mathrm{~mm})$. b A convex clockwise hysteresis loop develops when there is no relaxation at the end of lengthening and shortening.

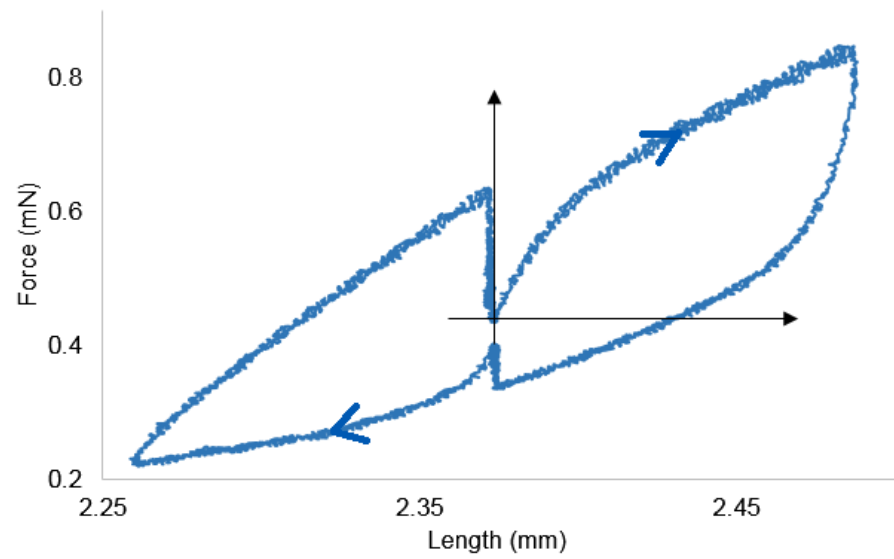

Fig. 12. Force-length relationship corresponding to Figure 10b. The curve consists of two loops that resemble those of kissand-go hysteresis (a concave clockwise hysteresis loop). The loops tend to touch one another at the origin, where Fo $=0.45$ $(\mathrm{mN})$ and $\mathrm{lo}=2.37(\mathrm{~mm})$ in this example.

frequency of stimulation than that used in Figure 13. All these four fibres had a length change of $5 \%$ lo. Only the first period of the force response is shown in each case.
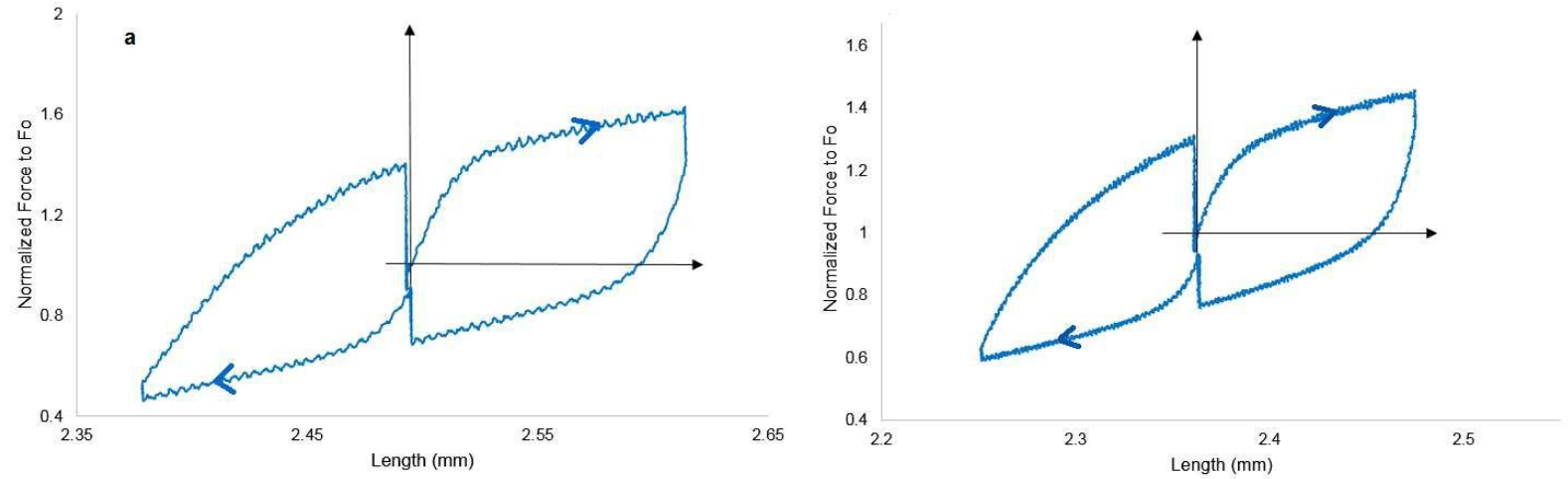

Fig. 13. Normalized force vs length for two different fibres a and $b$. The input frequency for both examples was $f=0.125$ $\left(s^{-1}\right)$. The force produced by the fibres reached different percentages of Fo.

The amount of force produced is different between the fibres even when it is normalized to Fo, as is 

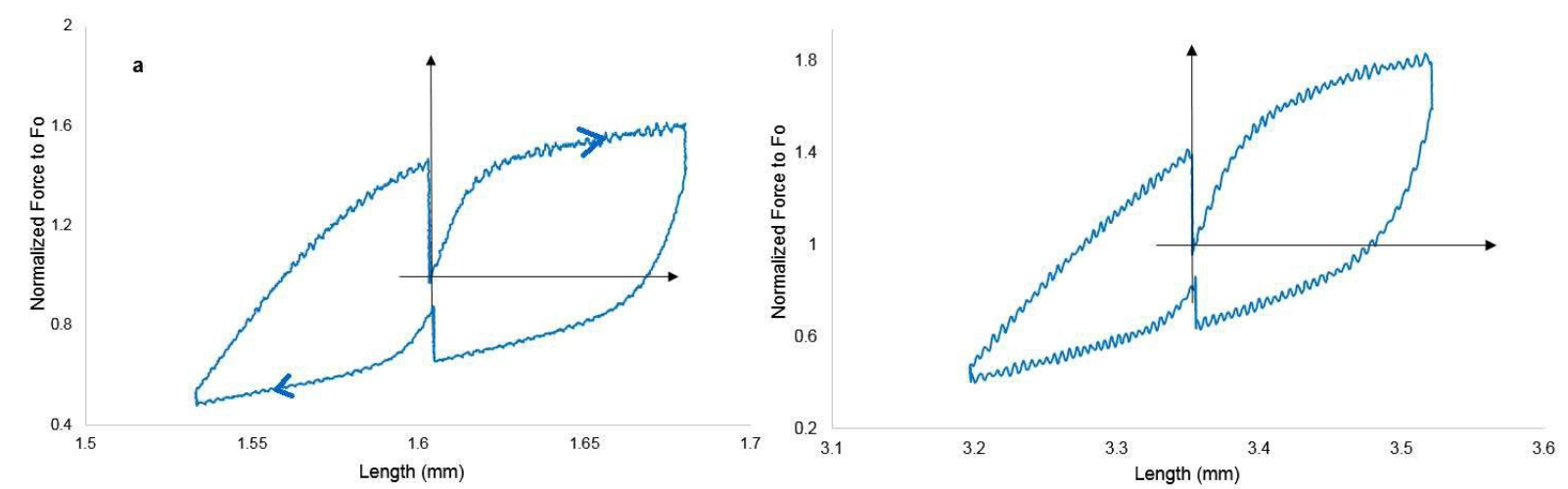

Fig. 14. Normalized force vs length for two different fibres. The input frequency was double that used to produce Figure 13 , $f=0.25\left(s^{-1}\right)$.

shown in Figures 13 and 14. However, the response pattern is similar, and therefore reproducible, between the fibres presented here.

\subsection{Force response to a $3 \%$ lo length change}

Examples of single fibres' force response to a length change of $3 \%$ lo are shown in Figures 15 and 16 , where the input frequency was $f=0.25\left(s^{-1}\right)$. In Figure 15, two periods of the output are plotted while in Figure 16, four periods of the force-length relationship are shown. A similar behavior was observed in all fibres, and in some cases (see below Figure 15b), the force response did not appear overlapped during the positive semi-periods. Both cycles of the force-length relationship appear overlapped in Figure 15a, while in Figure 15b the response to the positive semi-period (or right loop), appears as two loops not completely overlapped.
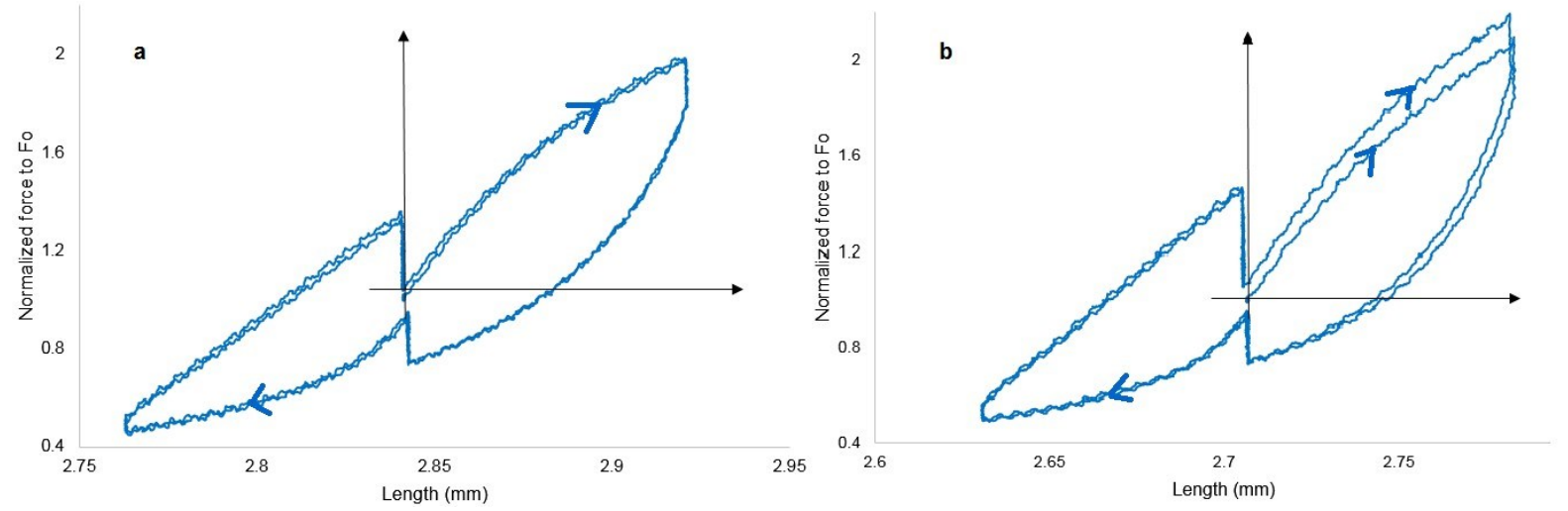

Fig. 15. Two cycles of length change and its normalized force response for two different fibres a and $b$ with $f=0.25\left(s^{-1}\right)$.

\subsection{Force response of a single fibre to different input frequencies}

In these experiments, the single muscle fibres were subjected to an input that varied its frequency from $f=0.125\left(\mathrm{~s}^{-1}\right)$ to $0.625\left(\mathrm{~s}^{-1}\right)$ to $1.25\left(\mathrm{~s}^{-1}\right)$. The objective of these experiments was to look at the shape of the force response (loops) from the same fibre when the input frequency had a two or ten-fold variation.

Figure 17a shows the hysteresis loops of a fibre subjected to a low frequency input, while the curve in Figure 17b shows the hysteresis loops of the same fibre for a two-fold increase of the input frequency (high frequency). For the second fibre, the curve in Figure 17c shows the loops at a low frequency and the curve in Figure 17d displays the hysteresis after a ten-fold increase in frequency. In both cases, the areas of the loops (equivalent to energy dissipated) increased with an increase of the input frequencies. 

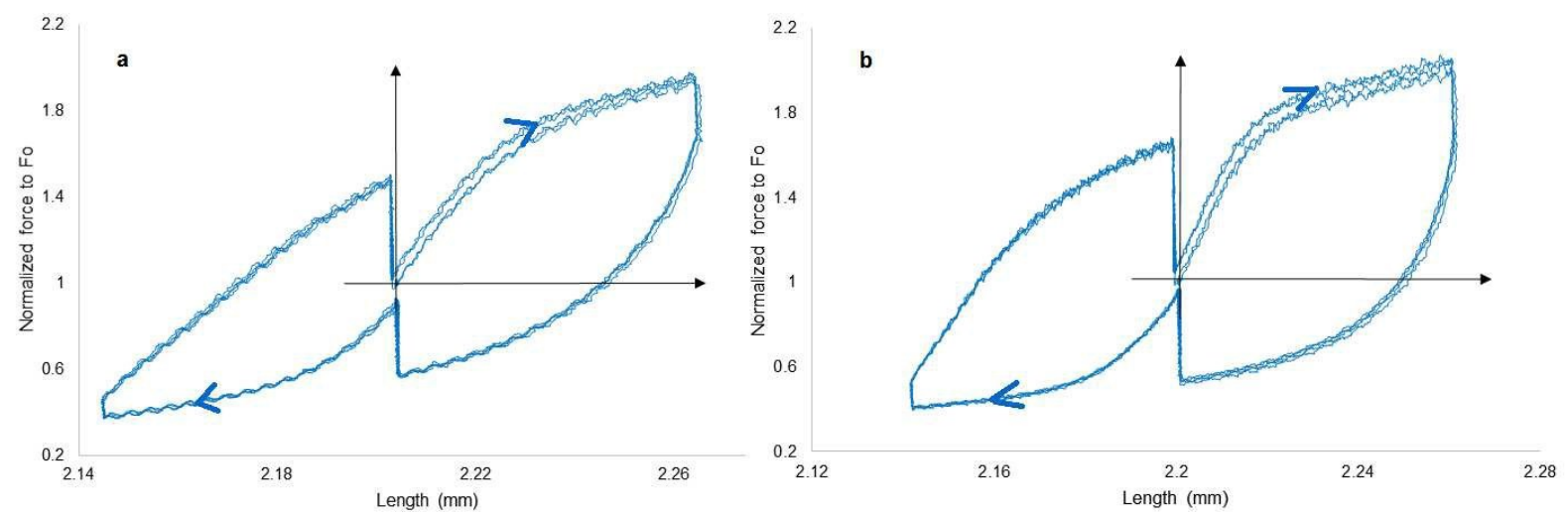

Fig. 16. Four cycles of normalized force vs length, for two different fibres a and b with $f=0.25\left(s^{-1}\right)$.
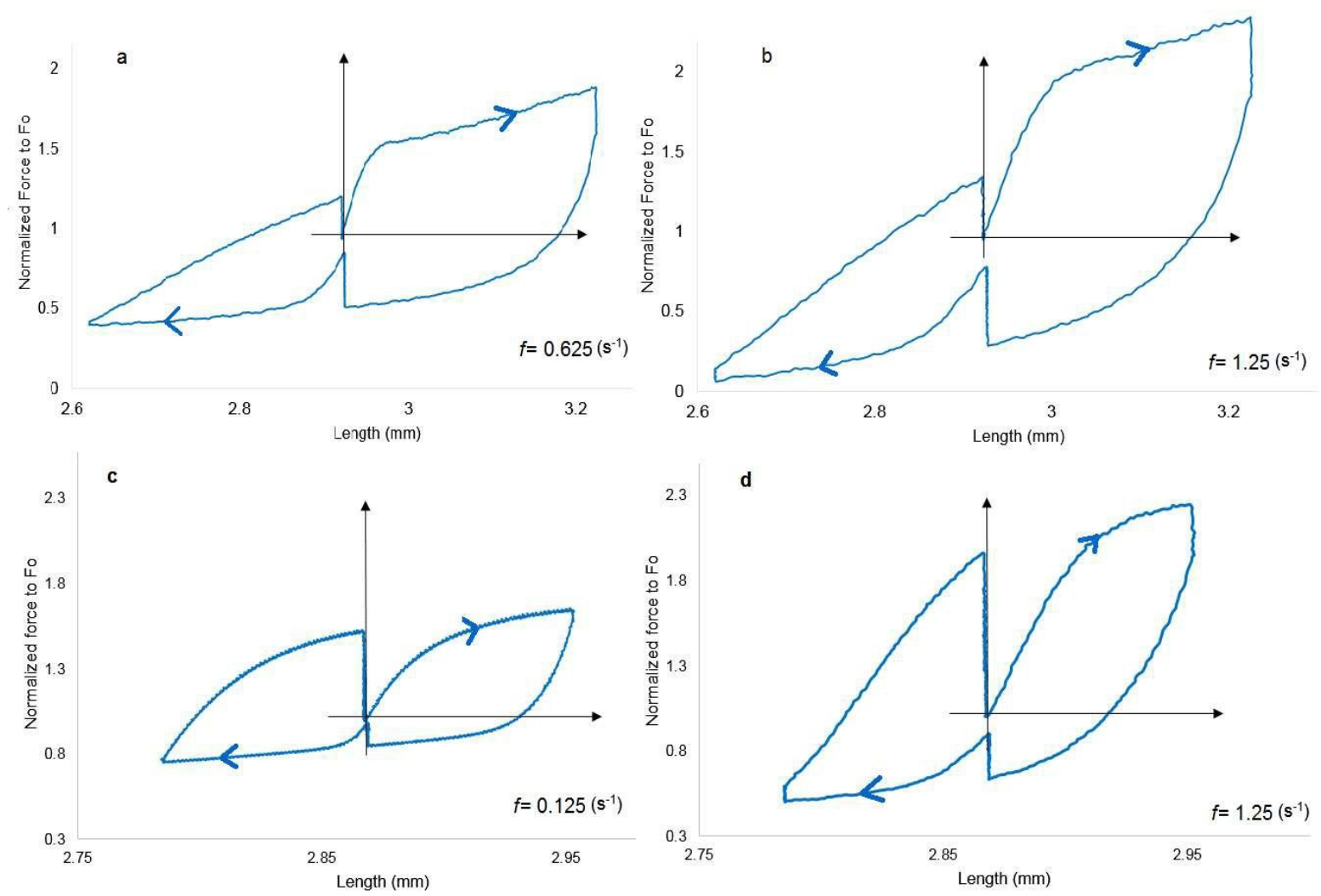

Fig. 17. Normalized force vs length for two single fibres. a a single fibre subjected to a low frequency input, $\mathbf{f}=0.625 \mathrm{~s}^{-1}$. The energy dissipated in this case was $3.98 \times 10^{-7} \mathrm{~J}$, and $\mathrm{b}$, the same fibre as used in a with $\mathbf{f}=1.25 \mathrm{~s}^{-1}$. The energy dissipated in this case was $5.88 \times 10^{-7} \mathrm{~J}$. c a single fibre subjected to a low frequency input, $f=0.125 \mathrm{~s}^{-1}$. The energy dissipated in this case was $8.45 \times 10^{-8} \mathrm{~J}$, and $d$, the same fibre as used in $c$ with $f=1.25 \mathrm{~s}^{-1}$. The energy dissipated in this case was $1.29 \times 10^{-7} \mathrm{~J}$. The amplitude change was $3 \%$ of lo in all cases.

\section{Mathematical model of lengthening and shortening}

\subsection{Hill's mathematical model}

Hill's seminal paper [Hill, 1938] published in 1938 presents detailed methods, experiments and mathematical modeling of muscle contraction. Hill employs classic macroscopic mechanics to represent the phenomenological muscle model. Figure 18 represents the viscoelastic model consisting of two elements in parallel with a series element. The contractile element $(C E)$ consists of a function $A(t)$ and a translational damper. 
This element is in parallel with a translational spring representing the parallel element (PE). Finally, the third element consists of a translational spring representing the series element (SE). The length of the parallel element is given by $L_{P E}$ and the length of the series element is given by $L_{S E}$. The length of the muscle is then $L_{M}=L_{P E}+L_{S E}$. The stiffness (relating changes in force with changes in length) of the translational springs 1 and 2 are given by $K_{P E}$ and $K_{S E}$, respectively. The same force $F$ develops to the left and right because the muscle can only have one force at any given time. Figure 18 is a Simscape ${ }^{T M}$ simulation model of a physical muscle system. Simscape enables one to rapidly create models of physical systems within the Simulink ${ }^{\mathbb{Q}}$ environment and is developed by MathWorks, Inc. Readers may also be interested in MapleSim which is a Modelica ${ }^{\mathbb{Q}}$-based system-level modeling and simulation tool developed by Maplesoft and Wolfram SystemModeler: a modeling and simulation environment.

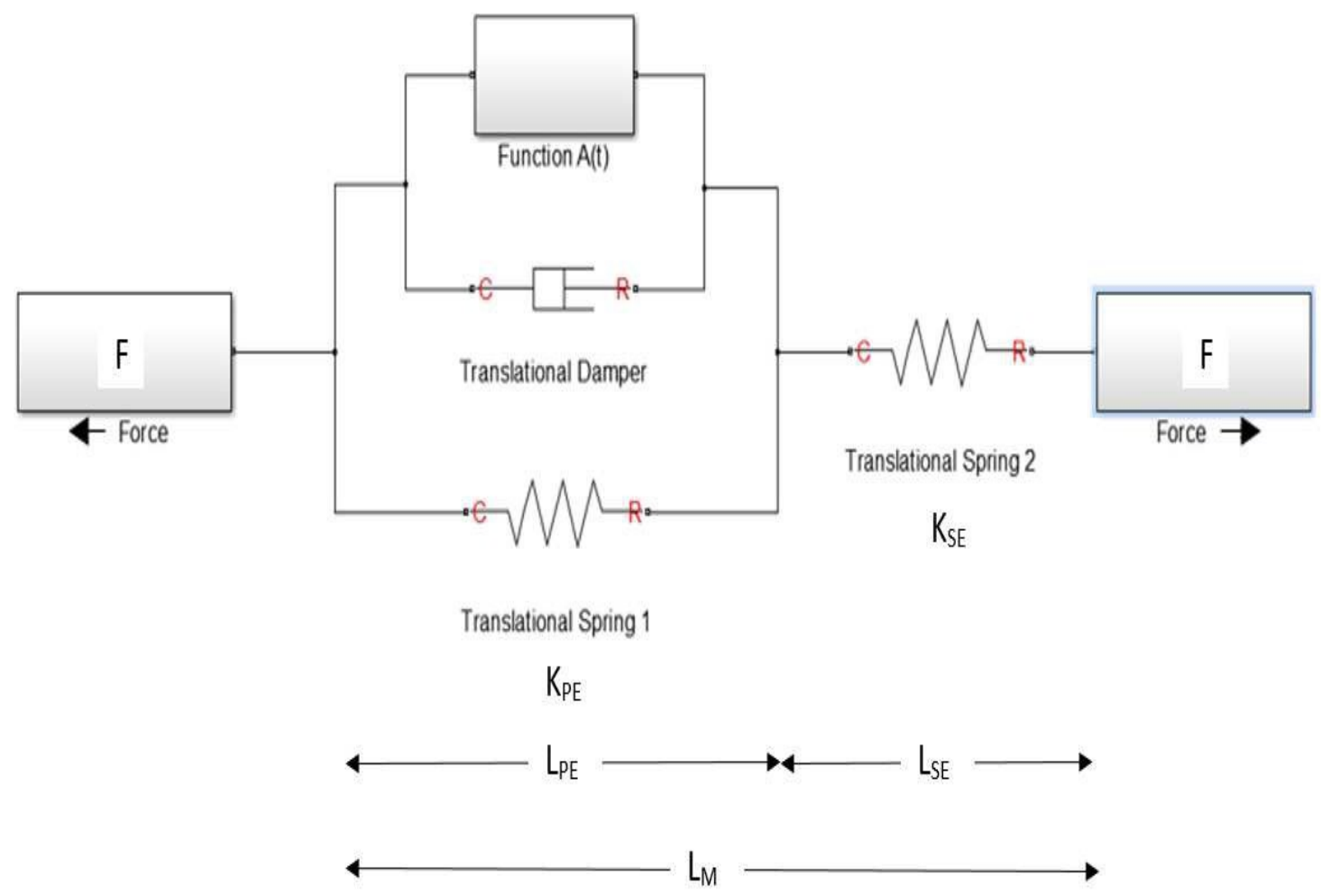

Fig. 18. A Simscape simulation of the Hill muscle model comprising of a series element (SE), a contractile element (CE) and a parallel element $(\mathrm{PE})$.

A simple differential equation representing the model depicted in Figure 18 is given by:

$$
\frac{d F(t)}{d t}=\frac{K_{S E}}{v_{d}}\left(K_{P E} \Delta L_{M}(t)+v_{d} \frac{d L_{M}(t)}{d t}-\left(1+\frac{K_{P E}}{K_{S E}} F(t)+A(t)\right.\right.
$$

where $A(t)$ is the active force, $v_{d}$ is viscosity in the damper, $\Delta L_{M}$ is the change in muscle length and $\frac{d L_{M}}{d t}$ is the speed of contraction of the muscle.

A simple MATLAB program of the Hill muscle model is described in [Holmes, 2006], where parameter values established by Hill are listed. This model was adapted to model lengthening and shortening muscle 
a

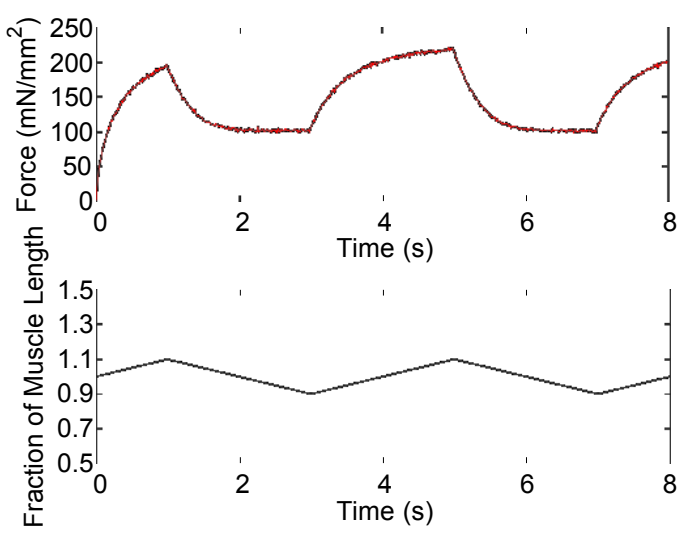

b

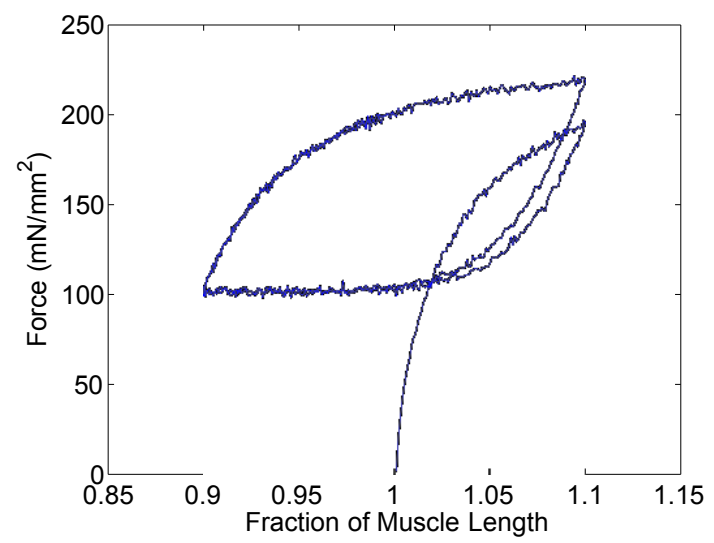

Fig. 19. a The lower figure shows lengthening and shortening as a fraction of muscle length against time. The upper figure shows the corresponding force response from the mathematical model. The parameters used in the model are the same as those listed in [Holmes, 2006]. b Force-length relationship of the mathematical model plotted using data from Figure 19a (compare with Figure 11b). Again there is a convex clockwise hysteresis loop when there is no relaxation at the end of lengthening and shortening. Some noise has been added to make the output look more realistic.

as described in the previous sections. The equations are given by:

$$
\begin{aligned}
L_{P E}(t) & =L_{M}(t)-L_{S E}(t), \\
L_{S E}(t) & =\frac{F(t)}{q}+L_{S E}(0), \\
\frac{d F(t)}{d t} & =\alpha \frac{d L_{M}(t)}{d t}+\frac{b(F(0)-F(t))}{a+F(t)},
\end{aligned}
$$

where $\alpha, a$ and $b$ are constants. Note that the equations for heat liberation are also listed in the paper but they are not required for the work in this paper. The parameters derived by experimentation by Hill [Holmes, 2006] and applied in equations (8) are given by $a=37.24 \mathrm{mN} / \mathrm{MM}^{2}, b=0.325$ lengths $/ \mathrm{sec}$, $\mathrm{F}(0)=\frac{a}{0.257} \mathrm{mN} / \mathrm{mm}^{2}, \alpha=\frac{F(0)}{0.1} \mathrm{mN} / \mathrm{mm}^{2}$ and $\mathrm{L}_{\mathrm{SE}}(0)=0.3$ (initially $30 \%$ of muscle length), and these are the parameters used in the models described in this paper.

\subsection{Results of the mathematical model}

Figure 19a shows the output of the mathematical simulation for a periodic lengthening and shortening without any relaxation. The lower graph displays the periodic fraction of muscle length change while the upper graph shows the force response. Using these results, Figure 19b displays the force-length relationship and a convex clockwise hysteresis loop is clearly visible. Notice the similarity of this loop to that shown by experiment in Figure 11.

Figure 20a shows the output of the mathematical simulation for a periodic lengthening and shortening with a relaxation phase at the end of each contraction. The lower graph displays the periodic fraction of muscle length change while the upper graph shows the force response. Using these results, Figure 20b displays the force-length relationship and a kiss-and-go hysteresis loop is clearly visible. Notice the similarity of this loop to those shown by experiments in Figures 12 to 17.

The classic macroscopic Hill model, consisting of simple first order ordinary differential equations, is sufficient to simulate contracting muscle when considering hysteresis effects and has been firmly established as a reliable model of muscle contractile dynamics for many decades. A review of the developments of the Hill model up to 2016 can be found in [Ovesy, et al., 2016]. One of the major disadvantages of the Hilltype models is that the viscoelastic analogies do not map to the underlying physiological mechanisms associated with muscle. Another popular mathematical model of muscle was proposed by Andrew Huxley in 1957 [Huxley, 1957] who introduced sliding filament theory and the cross-bridge cycle, where actin 
(thin) filaments slide past myosin (thick) filaments which attach and detach during muscle contraction. A comparison between mechanical behaviour modeled by Hill's equations and metabolic bahaviour modeled by Huxley's equations on muscle-tendon complex models using experimental data obtained from rat $\mathrm{m}$. soleus in situ is given in a recent paper [Lemaire el al., 2016]. Readers may also be interested in the work of Zahalak and Ma [Zahalak \& Ma, 1990] who bridge the gap between macroscopic and molecular muscle mechanics.

a

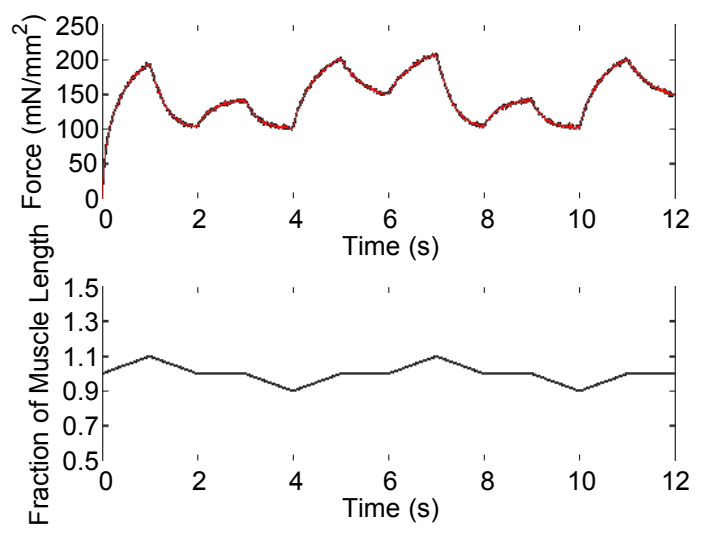

b

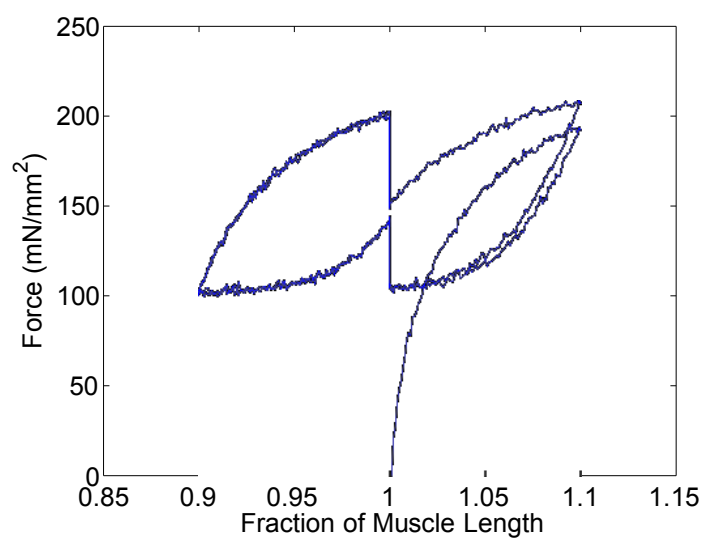

Fig. 20. a The lower figure shows lengthening and shortening as a fraction of muscle length against time. Note that there is relaxation at the end of each contraction. The upper figure shows the corresponding force response from the mathematical model. b Force-length relationship of the mathematical model plotted using data from Figure 20a (compare with Figures 12 to 17). In this case there are two loops that resemble those of kiss-and-go hysteresis. Some noise has been added to make the output look more realistic.

\section{Discussion}

The paper presents both a physical and mathematical model of single fibre lengthening and shortening and the force-length hysteresis cycles demonstrate that there is a history associated with the process. What is truly remarkable is that the mathematical models developed by Hill in the 1930s are still producing accurate representations of experimental data today.

Experimentally, the muscle force response to a periodic length change has been observed for different amplitude and frequency values of the input. Without relaxation, the force-length relationship consisted of a convex hysteresis loop for a bipolar triangle input. However, when a relaxation time was added at the end of each semi-period, the muscle fibres were able to return to their isometric state giving two characteristic hysteresis loops that have been reproducible in different experiments. The curves presented in Figures 12 to 17 show one loop on the right of lo representing the change in force when the fibre is lengthened and back to the initial length. Another loop is on the left of lo and represents the force change when the muscle fibre is shortened and returned to the initial fibre length. Although the loops are not completely closed, they are visibly separated. The behavior of this force-length relationship could be considered as "kiss-and-go" loops, as defined by [Volkov et al., 2014], where the branches do not self-cross, but tend to touch each other at the origin of the coordinates system, i.e. where $I=I 0$ and $F=F o$ for the curves in this study. This characteristic has been present in all the results regardless of the amplitude or frequency of the stimulation. Furthermore, the area bounded by each loop represents the energy dissipated in each cycle of lengthening and shortening. In some of the examples, the energy dissipated in the right loop is higher than that dissipated in the left loop, for a similar amount of work done on or by the muscle (lengthening or shortening). This difference in the loop size shows that a higher amount of energy is converted into heat, meaning that the energy conversion to work is less efficient. It appears that the energy stored into the stretching muscle beyond optimal length can be used during subsequent shortening, making cyclical 
movements, such as walking and running more economical than if this was not the case.

In this study, two different amplitudes of the length change were evaluated, 5 and $3 \%$ lo. The observed force response as a \% Fo was independent of the amplitude. In Figures 15 and 16 are shown two and four response cycles, respectively. The hysteresis loops on the right of Figures 15b,16a and 16b do not appear completely overlapped for the whole duration of the experiment. Particularly, in Figure 15b, the force produced in the second cycle (at the end of the stretch) was higher than that one in the first cycle, given that the force starting point for the second cycle was somehow higher than that from the start of the first period. This occurs as a result of muscle fibre stretching and being brought back to lo where it did not relax completely to Fo after a complete period of length change, but to a higher force value. Despite the force production differing between different single fibres, the force versus length relationship consisted of two hysteresis loops that tend to touch each other at the origin of the coordinate system.

When the muscle single fibre was subjected to different input frequencies, the area of the loops increased as did the stimulation frequency by two or ten-fold (Figure 15). The change in response when the input frequency changed means that this system has rate dependent hysteresis.

Hill's macroscopic mathematical model was employed using the parameters listed in his paper and the same characteristic hysteresis loops are generated as those shown from experiment. Future research could focus on muscle fatigue, and the metabolic Huxley [Huxley, 1957] and macroscopic/metabolic Zahalak and Ma [Zahalak \& Ma, 1990] mathematical models subject to periodic contractions.

In conclusion, when a muscle single fibre is considered as a mechanical system and subjected to a periodic input, the force vs length relationship showed hysteresis loops and the system was rate-dependent. These loops were reproducible both experimentally and with mathematical modeling and the hysteresis observed in this study is not a pinched hysteresis, but is labeled here as kiss-and-go. Future research is expected to concentrate on other forms of hysteresis in muscle, and another potential avenue of research is in critical slowing down in muscle, where the return time of a disturbance back to a stable steady-state increases close to a bifurcation, see [Bardy et al., 1999] and [Alharbey et al., 2014], for example.

\section{Acknowledgements}

This research was funded by the European Commission through MOVE-AGE an Erasmus Mundus Joint Doctorate program (2011-2015) and an EPSRC Nano-Info-Bio project through Manchester Metropolitan University. We would like to take this opportunity to thank the referees for their extremely useful comments.

\section{References}

Ewing, J.W. [1885] “Experimental researches in magnetism”, Transactions of the Royal Society of London $176,523640$.

Piramanayagam, S.N. and Chong, T.C. [2011] Developments in Data Storage: Materials Perspective, (Wiley-IEEE Press).

Muller, I. and Xu, H. [1991] "On the pseudo-elastic hysteresis," Acta Metallurgica et Materialia, 39, 263271.

Smith, R.C., Seelecke, S., Dapino, M., et al. [2006] "A unified framework for modeling hysteresis in ferroic materials," Journal of the mechanics and physics of solids, 54, 46-85.

Damjanovic, D. [2005] "Hysteresis in piezoelectric and ferroelectric materials," The Science of Hysteresis 3, eds. Mayergoyz, I and Bertotti, G., (Elsevier), 337-465.

Lynch, S. and Steele, A.L. [2011] "Nonlinear optical fibre resonators with applications in electrical engineering and computing," Applications of Nonlinear Dynamics and Chaos in Engineering eds. Banerjee, S., Mitra, M. and Rondoni, L. (Springer) 1, 65-84.

Lynch, S. [2011] "MATLAB programming for engineers and scientists," Applications of Nonlinear Dynamics and Chaos in Engineering. eds. Banerjee, S., Mitra, M. and Rondoni, L., (Springer) 1, 3-35.

Lynch, S., Alharbey, R.A., Hassan, S.S. and Batarfi, H.A. [2015] "Delayed-dynamical bistability within 
and without rotating wave approximation," Journal of Nonlinear Optical Physics and Materials, 24, 1550037.

Borresen, J. and Lynch, S. [2002] “Further investigation of hysteresis in Chua's circuit," Int J of Bifurcation and Chaos 12, 129-134.

Lynch, S. and Christopher, C.J. [1999] "Limit cycles in highly non-linear differential equations," J Sound and Vibration 224 505-517.

Ball, L.M. [2009] "Hysteresis in Unemployment: Old and New Evidence," US National Bureau of Economic Research (NBER), Working Paper No. 14818.

Lynch, S. [2005] "Analysis of a blood cell population model," Int J of Bifurcation and Chaos 15, 2311-2316.

Walcott S. and Sun X. [2009] "Hysteresis in cross-bridge models of muscle," Phys. Chem. Chem. Phys. 11, 4871-4881.

Lynch, S. and Bandar, Z. [2005] "Bistable neuromodules," Nonlinear Anal Theory, Meth. \& Appl. 63, 669-677.

Pomerening, J.R., Sontag, E.D., Ferrell, J.E. [2003] "Building a cell cycle oscillator: hysteresis and bistability in the activation of Cdc2," Nature Cell Biology 5, 346251.

Kramer, B.P., Fussenegger, M. [2005] "Hysteresis in a synthetic mammalian gene network," Proceeding of the National Academy of Sciences USA, 102, 9517-9522.

Das, J., Ho M., Zikherman, J., Govern, C., Yang, M., Weiss, A, Chakraborty, A.K., Roose, J.P. [2009] “Digital Signaling and Hysteresis Characterize Ras Activation in Lymphoid Cells," Cell 136, 337351.

Lynch, S., Borresen, J. [2015] "Oscillations, feedback and bifurcations in mathematical models of angiogenesis and haematopoiesis," Handbook of Vascular Biology Techniques, eds. Slevin M, McDowell G, Cao Y, Kitajewski J., (Springer, New York), 373-390.

Noori, H.R. [2014] Hysteresis Phenomena in Biology, (Springer Berlin Heidelberg).

Lynch, S. [2014] Dynamical Systems with Applications using MATLAB 2nd ed., (Springer International Publishing).

Lynch, S. [2010] Dynamical Systems with Applications using Maple 2nd ed., Springer, New York.

Lynch, S. [2007] Dynamical Systems with Applications using Mathematica, Springer, New York.

Drincic, B. and Bernstein, D.S. [2011] “Why are some hysteresis loops shaped like a butterfly?” Automatica, 47, 2658-2664.

Thompson J.M.T. and Stewart H.B., Nonlinear Dynamics and Chaos (2nd edition), John Wiley \& Sons, 2002.

Ott E., Chaos in Dynamical Systems (2nd edition), Cambridge University Press, 2002.

Ikeda, K. [1979] "Multiple-valued stationary state and its instability of the transmitted light by a ring cavity system," Opt. Comm. 30, 257261.

Ikeda, K., Daido, H. and Akimoto, A. [1980] "Optical turbulence: chaotic behavior of transmitted light from a ring cavity,” Phys. Rev. Lett. 45, 709712.

Lynch, S., Steele, A.L. and Hoad, J.E. [1998] "Stability analysis of nonlinear optical resonators," Chaos, Solitons and Fractals, 9, 935-946.

Rahatabad, F.N., Fallah, A., Jafari, A.H. [2011] "A study of chaotic phenomena in human-like reaching movements," International Journal of Bifurcation and Chaos 21, 3293-3303.

Webber C.L. and Zbilut J.P. [1994] "Dynamical assessment of physiological systems and states using recurrence plot strategies," Journal of Applied Physiology 76, 965-973.

Weiss, J.N., Garfinkel, A., Karagueuzian, H.S., Qu, Z.L., Chen, P.S. [1999] "Chaos and the transition to ventricular fibrillation - A new approach to antiarrhythmic drug evaluation," Circulation 99, 28192826.

Gauthier, D.J., Hall, G.M., Oliver, R.A., Dixon-Tulloch, E.G., Wolf, P.D., Bahar, S. [2002] “Progress toward controlling in vivo fibrillating sheep atria using a nonlinear-dynamics-based closed-loop feedback method," Chaos 12, 952-961.

Kyriatzis M. [2003] "Practical applications of chaos theory to the modulation of human ageing: nature prefers chaos to regularity," Biogerontology 4, 7590.

Szöke, A., Daneu, V., Goldhar, J. and Kirnit, N.A. [1969] "Bistable optical element and its applications," Appl. Phys. Lett. 15, 376. 
Hodgkin, A.L. and Huxley, A.F. [1952] "A qualitative description of membrane current and its application to conduction and excitation in nerve," Journal of Physiology 117, 500-544.

Qingjiang, L., Khiat, A. et al. [2014] "Memory Impedance in TiO2 based Metal-Insulator-Metal Devices," Scientific Reports 4, Article number: 4522.

Chua, L.O. [1971] "Memristor-missing circuit element," IEEE Transactions on Circuit Theory CT18, 507519.

Chua, L.O., and Kang, S.M. [1976] “Memristive devices and systems," Proceedings of the IEEE 64, 209-223.

Prodromakis, T., Toumazou, C. and Chua, L.O. [2012] “Two centuries of memristors," Nat. Mater. 11, 478-481.

Chua, L.O. [2013] “Memristor, Hodgkin-Huxley and the edge of chaos," Nanotechnology 24, 383001.

Mayergoyz, I.D. [2003] "Mathematical Models of Hysteresis and Their Applications," Academic Press Series in Electromagnetism, (Elsevier).

Volkov, A.G., Tucket, C., Reedus, J., Volkova, M. I., Markin, V.S. and Chua, L.O. [2014] "Memristors in plants," Plant Signaling and Behavior, 9, 18.

Chua, L.O. [2014] "If its pinched its a memristor." Semiconductor Science and Technology, 29, 104001.

Gilliver, S.F., Jones, D.A., Rittweger, J. and Degens, H. [2010] "Effects of oxidation on the power of chemically skinned rat soleus fibres," Journal of Musculoskeletal and Neuronal Interactions, 10, 267273.

Degens, H., Bosutti, A., Gilliver, S.F., Slevin, M., Van Heijst, A. and Wust, R.C.I. [2010] "Changes in contractile properties of skinned single rat soleus and diaphragm fibres after chronic hypoxia," European Journal of Physiology, 460, 863-873.

Gilliver, S.F., Degens, H., Rittweger, J. and Jones, D.A. [2011] "Effects of submaximal activation on the determinants of power of chemically skinned rat soleus fibres." Experimental Physiology, 96, 171-178.

Larsson,L. and Moss, R. L. [1993] "Maximum velocity of shortening in relation to myosin isoform composition in single fibres from human skeletal muscles," Journal of Physiology, 472, 595-614.

Gilliver, S.F., Degens, H., Rittweger, J., Sargeant, J. and Jones, D.A. [2009] "Variation in the determinants of power of chemically skinned human muscle fibres," Experimental Physiology, 94, 1070-1078.

Lahaye, M. and Rochas, C. [1991] "Chemical structure and physico-chemical properties of agar," Hydrobiologia, 221, 137-148.

Armisen, R. and Galatas, F. [1987] "Production, properties and uses of agar," FAO, 137-148.

Hill, A.V. [1938] "The heat of shortening and the dynamic constants of muscle," Proc. Roy. Soc. B, 126, 136-195.

Holmes, J.W. [2006] “Teaching from classic papers: Hill's model of muscle contraction,” Adv. Physiol. Educ. 30, 67-72.

Ovesy, M., Nazari, M.A. and Mahdavian, M. [2016] "Equivalent linear damping characterization in linear and nonlinear force-stiffness muscle models," Biol. Cybern. 110, 73-80.

Huxley, A.F. [1957] "Muscle structure and theories of contraction," Proc. Biophys. and Biophys. Chem., 7, 257-318.

Lemaire, K.K. Baan, GC., Jaspers, R.T. and van Soest, A.J. [2016] "Comparison of the validity of Hill and Huxley muscle-tendon complex models using experimental data obtained from rat $\mathrm{m}$. soleus in situ," J. Experimental Biol. 129, 977-987.

Zahalak, G.I and Ma, S.P. [1990] "Muscle activation and contraction: constitute relations based directly on cross bridge kinetics," J. Biomech. Eng. 112, 52-62.

Bardy, B.G., Oullier, O., Bootsma, R.J., Stoffregen, T.A. [1999] “Dynamics of human postural transitions," Journal of Experimental Psychology-Human Perception and Performmance, 28, 499-514.

Alherbey, R.A., Nejad, L.A.M., Lynch, S. and Hassan, S.S. [2014] "Critical slowing down in biological bistable models," International Journal of Pure and Applied Mathematics, 93, 581-602. 